\title{
LABORATORY DISPERSIVE EXAFS SPECTROMETER
}

\author{
A. Mosset, P. Lecante, P. Baules, J. Jaud, J. Galy \\ CEMES-CNRS, 29 rue Marvig, BP 4347, 31055 Toulouse, France
}

\author{
AND A. BURIAN \\ Institute of Engineering Problems, University of Silesia \\ Śnieżna 2, 41-200 Sosnowiec, Poland
}

\begin{abstract}
We report on a laboratory EXAFS spectrometer optimized for transmission dispersive mode. Absorption edges ranging from 6 to $20 \mathrm{keV}$ can be studied. Factors determining energy range and resolution are detailed. A new asymmetric geometry which allows to use all the lattice planes of the dispersing crystal is described and the choice of the best plane analysed. EXAFS spectra of $\mathrm{Cu}$ metal and $\mathrm{Cu}$ acetate, obtained on this laboratory facility, are compared to the corresponding spectra recorded on the French synchrotron facility (LURE).
\end{abstract}

PACS numbers: $61.10 . \mathrm{Ht}, 07.85 . \mathrm{Nc}, 61.43 .-\mathrm{j}$

\section{Introduction}

The study of short-range order in liquids, solutions and non-crystalline solids usually involves wide angle X-ray scattering (WAXS) and/or EXAFS measurements. Synchrotron radiation constitutes the best source currently available for EXAFS experiments. However, the accessibility to the large storage rings is sometimes difficult. Thus various laboratory facilities using conventional X-ray tubes were developed in order to perform routine EXAFS measurements in all studies where a high sensitivity is not required.

Most of existing in-lab spectrometers use bent monochromator and work in sequential mode. They require frequent tedious adjustments and very accurate mechanical motions during the experiment. An alternative technique is the dispersive mode which is based on the divergent character of the beam produced by $\mathrm{X}$-ray tubes. X-rays falling on a single crystal settled in transmission or reflection geometry with different incidence angles are diffracted for different Bragg related energies. 


\section{Principle and realization}

In the transmission dispersive method [1-3], the divergent beam emitted by a conventional X-ray tube is diffracted by a family of lattice planes of a thin Si single crystal. The diffracted beam converges on the sample located at the focus. Behind the sample, $\mathrm{X}$-rays of different energies are dispersed and the linear position sensitive detector is moved to a distance from the sample which allows to record the whole intensity spectrum assumed to be $1000 \mathrm{eV}$ in width. All data points are simultaneously recorded. Mechanical motions are thus suppressed during data collection reducing potential drift and background and allowing long exposures.

The energy range covered for a fixed central energy $E_{0}$ is given by the equation $\left(E_{\max }-E_{\min }\right) / E_{0}=\alpha \cot \theta_{0}$, where $\alpha$ is the angular divergence of the incident beam and $\theta_{0}$ - the angle between the central incident beam and the lattice plane. The energy resolution can be calculated as

$$
\Delta E / E_{0}=\left[\left(s / l_{\mathrm{sc}}\right)^{2}+\left(w / l_{\mathrm{sc}}\right)^{2}+\left(r / l_{\mathrm{sd}}\right)^{2}+\omega^{2}+\phi^{4} / 4 \tan ^{2} \theta_{0}\right]^{1 / 2} \cot \theta_{0},
$$

where $s$ is the horizontal width of the X-ray source, $w=2 t \sin \theta_{0}$ - the spatial broadening of the beam for a dispersing crystal of thickness $t, r$ - the spatial resolution of the detector, $l_{\mathrm{sc}}$ and $l_{\mathrm{sd}}$ - the distances from source to crystal and from sample to detector, $\omega$ - the intrinsic angular width of the crystal and $\phi-$ the vertical divergence of the beam. The dispersing crystal is a $3^{\prime \prime}$ silicon wafer cut along the [001] axis. It is chemically thinned down to a thickness between $50 \mu \mathrm{m}$ and $100 \mu \mathrm{m}$. The different lattice planes can be selected by a rotation of the crystal holder perpendicular to the crystal surface. Two geometries can be used for the measurements depending on the selected plane, a symmetric geometry when using the "even" planes, e.g. (220) or (400), or an asymmetric geometry when using the "odd" planes, e.g. (111) or (311). The "odd" diffraction planes are particularly interesting in order to get higher fluxes and thus shorter measurement times by increasing the voltage $V$ of the X-ray tube because they do not present harmonics if $V$ is smaller than $3 E$ instead of $2 E$ ( $E=$ edge energy). Doing this, a large range of energy, 6 to $20 \mathrm{keV}$, is accessible which corresponds to the $K$ edges from vanadium to molybdenum and the $L$ edges from cerium to bismuth.

A single family of diffraction planes does not permit to cover the whole energy range. Figure 1a shows the variation of the theoretical resolution $\Delta E / E_{0}$ with the energy for the Si diffraction planes. For each family, only the linear part of the curve is given; for higher energies, the resolution deteriorates very rapidly. In the equation giving $\Delta E / E_{0}$, the second and third terms which involve the thickness $t$ of the dispersing crystal and the resolution of the detector are predominant.

The thickness $t$ also influences the integrated energy in a complex manner. For a thin and weakly absorbing perfect crystal, the equations of the dynamical theory of X-ray diffraction show that the diffraction profiles exhibit strong oscillations [4]. In order to evaluate this effect, the method from Ref. [4] was used to integrate the diffracted intensity on the angular domain. Figure $1 \mathrm{~b}$ shows the result of this calculation for the Si diffraction planes taking into account the actual divergence accepted by the detector and the intensity loss caused by the vertical divergence of the diffracted beam. Obviously, the shapes of the intensity curves on the whole energy range are very complex. A comparison of the relative intensities 

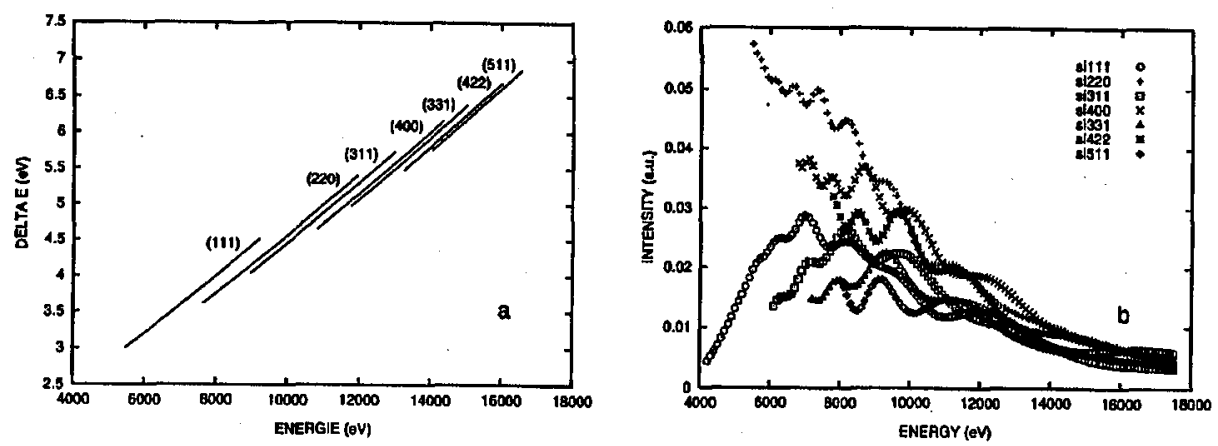

Fig. 1. (a) Theoretical resolution for the Si diffraction planes; (b) integrated intensity of diffraction profiles for Si planes corrected for the horizontal beam divergence and the $l_{\text {sd }}$ distance (thickness $t=100 \mu \mathrm{m}$ ).

leads to the conclusion that the (220) and (400) are the best choice. However, the (111) plane allows to work with a higher voltage of the X-ray tube if the detector cannot discriminate the harmonics and constitutes also a good choice for the low energies.

A small value of the crystal thickness allows to smooth these intensity variations and to get a high intensity over a wide energy range.

\section{Results}

EXAFS spectra were collected for a copper foil and a pellet of copper acetate using the described instrument and the EXAFS III spectrometer at the French synchrotron facility LURE.

The copper foil was $10 \mu \mathrm{m}$ thick. The sample of copper acetate was pelleted by pressing a mixture of a finely powdered $\mathrm{Cu}\left(\mathrm{CH}_{3} \mathrm{COO}\right)_{2}$ and an organic diluting medium (glyoxal hydrate). Measurement times for each sample were $20 \mathrm{~min}$ at LURE and $24 \mathrm{~h}$ on the lab facility using the (220) planes. A complete analysis of
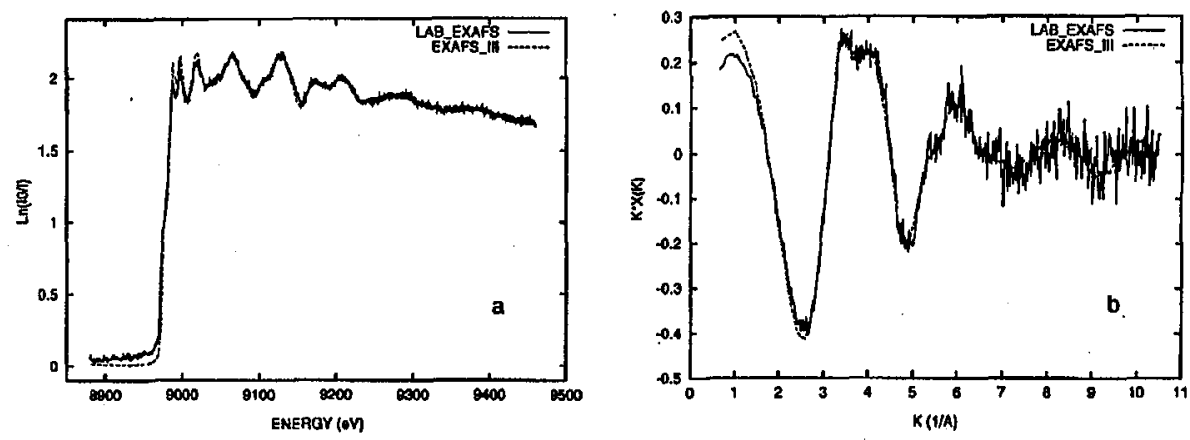

Fig. 2. (a) Laboratory EXAFS and EXAFS III spectra for a copper foil; (b) $k \chi(k)$ functions for copper acetate. 
the data sets was performed using the same standard procedures for the LURE and the laboratory spectra.

For the two samples, both measurements are remarkably close in shape. As shown in Fig. 2a, fine details observed for the copper foil just above the absorption edge prove that the effective resolution of the laboratory facility is, in that case, close to the EXAFS III resolution and to the theoretical one. As expected, the background is significantly smaller for the synchrotron measurements. However, even if it is noisy, the $k \chi(k)$ function obtained for copper acetate (Fig. 2b) on the laboratory facility is remarkably similar to the LURE function.

\section{Conclusion}

This optimization study of a laboratory EXAFS spectrometer working in transmission dispersive mode shows that high quality spectra can be obtained on such an instrument. One of the key factor is the thickness and the quality of the dispersing crystal. In order to get a high diffracted intensity and a high resolution, the thickness and the selected lattice plane must be optimal. This spectrometer could be still more improved using recently developed solid-state detectors.

\section{References}

[1] U. Kaminaga, T. Matushita, K. Kohra, Jpn. J. Appl. Phys. 20, L355 (1981).

[2] R. Buschert, M.D. Giardina, A. Merlini, A. Balerna, S. Mobilio, J. Appl. Crystallogr. 21, 79 (1988).

[3] P. Lecante, J. Jaud, A. Mosset, J. Galy, A. Burian, Rev. Sci. Instrum. 65, 845 (1994).

[4] M. Sanchez del Rio, C. Ferrero, G.J. Chen, F. Cerrina, Nucl. Instrum. Methods Phys. Res. A 347, 338 (1994). 